\title{
A muon simulation for the AMoRE-II experiment
}

\author{
Hanwook Bae* \\ Department of Physics, Kyungpook National University, Daegu, Korea \\ E-mail: klpp5588@naver.com
}

\section{Eunju Jeon}

Center for Underground Physics, Institude for Basic Science, Daejeon, Korea

E-mail: ejjeon@ibs.re.kr

\section{Sehwook Lee}

Department of Physics, Kyungpook National University, Daegu, Korea

E-mail: seh.wook. lee@cern.ch

The AMoRE-II is an experiment to search neutrino-less double beta decay of Mo-100 which is the phase II of the AMoRE experiment. If we found the double beta decay, it means that the neutrinos are Majorana particles and we can measure their masses. We studied effects of neutrons and gammas which are induced by cosmic muons. Detailed results with discussions will be shown on the poster.

The 39th International Conference on High Energy Physics (ICHEP2018)

4-11 July, 2018

Seoul, Korea

${ }^{*}$ Speaker. 


\section{Motivation}

The experiment is going to be carried out in the deep underground to observe the extremely rare events free from the backgrounds coming from the cosmic ray particles. However, some cosmic ray particles like muons can affect the signal measurement. Therefore, we must exclude the effects from the muons as much as possible. The motivation of this simulation is to provide an estimation of the background event rate by muon and its secondaries, and detailed information about the background events.

\section{Simulation configuration}

First of all, we installed the lead in the shape of a cylinder to surround the CMO crystal in the simulation. Then, we also placed the plastic scintillator at the outside of the lead shield to detect charged particles. The muons for this simulation has been generated with a contour of the Mt. Yemi. The generated muons are corresponding to the interval of 9.17 years.

\section{Energy deposit on scintillator}

The gammas and neutrons from muons and muon itself make the background in the CMO crystals. The peak and bumps are due to geometry structure of the scintillator. We have set the veto threshold to be $7.5 \mathrm{MeV}$ to veto the most of the muons.

\section{The background rate from gamma and neutron}

The background rate from neutrons does not much change as increasing of the thickness of lead. Meanwhile, the background event by gammas decreases dramatically as increasing of the lead shield thickness. The most of gammas can be prevented with $30 \mathrm{~cm}$ of lead. Thus, we continued the simulation analysis with $30 \mathrm{~cm}$ of lead.

\section{The energy deposit in CMO crystals with $30 \mathrm{~cm}$ lead shield and incident neutrons}

The background rate in the range of interest is $7.44 \times 10^{-6}$, most of them has been created by incident neutrons. The mean kinetic energy of neutrons is $43.55 \mathrm{MeV}$ and 503 neutrons have the kinetic energy of below than $1 \mathrm{MeV}$.

\section{Summary}

- If we can veto the most of the muons, then the most of background events come from gammas and neutrons.

- Also, we can shield the most of the gammas with $30 \mathrm{~cm}$ thickness of the lead shield.

- The remaining particle, neutrons have the mean kinetic energy of $43.55 \mathrm{MeV}$.

- The veto performance can be improved by shielding those neutrons. Thus, we will perform the further simulation for neutron shielding. 